\title{
Learning curve of endorectal ultrasonography in preoperative staging of rectal carcinoma
}

\author{
ZUO-LIANG LIU ${ }^{1}$, TONG ZHOU ${ }^{1}$, XIAO-BO LIANG ${ }^{2}$, JUN-JIE MA ${ }^{2}$ and GUANG-JUN ZHANG ${ }^{1}$ \\ ${ }^{1}$ Department of Gastrointestinal Surgery, Institute of Hepatobiliary, Pancreas and Intestinal Disease, \\ The Affiliated Hospital of North Sichuan Medical College, Nanchong, Sichuan 637000; \\ ${ }^{2}$ Department of Anorectal Surgery, Shanxi Province Tumor Hospital, \\ Taiyuan, Shanxi 030001, P.R. China
}

Received May 22, 2014; Accepted June 17, 2014

DOI: $10.3892 / \mathrm{mco} .2014 .352$

\begin{abstract}
Accurate preoperative staging of rectal carcinoma is essential for optimal treatment. This study was designed to evaluate the accuracy and learning curve of endorectal ultrasonography (ERUS) in the preoperative staging of rectal carcinoma. We retrospectively analyzed the records of patients with rectal carcinoma who underwent preoperative ERUS followed by curative surgery at the Shanxi Province Tumor Hospital between January, 2007 and March, 2010. The patients were divided into three groups, namely A, B and C, depending on whether the examination was performed between January and December, 2007, between January and December, 2008 or between January, 2009 and March, 2010, respectively. Five physicians with no prior experience in ERUS performed the examinations. We compared the ERUS staging with the pathological findings using the tumor-node-metastasis (TNM) classification. The accuracy of ERUS in $\mathrm{T}$ and $\mathrm{N}$ staging after each additional consecutive 20 patients was calculated for physicians D, E and F. A total of 319 patients underwent ERUS prior to surgery. There were 38 patients in group A, 135 in group B and 146 in group C. Two of the five physicians performed only 47 of the 319 examinations, whereas the remaining 272 patients were examined by physicians $D$ $(n=162), E(n=64)$ and $F(n=46)$. The overall accuracy in assessing the extent of rectal wall invasion (T) was $67 \%$, with $16 \%$ of the cases overstaged and $17 \%$ understaged and the accuracy in assessing nodal involvement $(\mathrm{N})$ was $66 \%$, with $11 \%$ of the cases overstaged and $23 \%$ understaged. The total $\mathrm{T}$ and $\mathrm{N}$ staging accuracy of physicians $\mathrm{D}, \mathrm{E}$ and $\mathrm{F}$ was 75 and $72 \%$; 59 and 59\%; and 50 and 52\%, respectively. For
\end{abstract}

Correspondence to: Professor Tong Zhou, Department of Gastrointestinal Surgery, Institute of Hepatobiliary, Pancreas and Intestinal Disease, The Affiliated Hospital of North Sichuan Medical College, No. 63, Wenhua Road, Nanchong, Sichuan 637000, P.R. China E-mail: zhoutong0088@163.com

Key words: rectal carcinoma, endorectal ultrasonography, learning curve, staging, accuracy, tumor invasion physicians D, E and F, the accuracy of T and $\mathrm{N}$ staging after each additional 20 patients was calculated and the curve of the accuracy reached a plateau after physician D completed 80 cases. Therefore, ERUS is a valuable tool for assessing the depth of tumor invasion and it appears that after $\sim 80$ cases a physician may be considered able to apply it efficiently.

\section{Introduction}

Colorectal cancer is the third most common type of cancer and the second or third most common cause of cancer-related mortality worldwide. Approximately one-third of the tumors arise in the rectum, whereas in the remaining cases the tumor arises in the colon and the majority of the cases are carcinomas (1). The preoperative assessment of the depth of cancer invasion of the rectal wall and regional lymph node metastasis is crucial in determining the surgical approach for the treatment of rectal cancer.

Multiple modalities are available for the staging of rectal cancer, including digital examination, endorectal ultrasonography (ERUS), computerized tomography (CT), magnetic resonance imaging (MRI) and, more recently, positron emission tomography (PET)-CT. Clinical staging by digital examination is standard, but does not allow assessment of the nodal status. Although CT scanning is useful for assessing extramural tumor spread, it is less accurate in assessing the depth of invasion within the wall or in predicting nodal disease. The accuracy of MRI and PET-CT were found to be higher, but are more costly. ERUS, being a non-invasive modality, has been proven to be fast, safe, cost-effective, efficient and is currently a widely used diagnostic tool in the assessment of the depth of cancer invasion and lymph node involvement $(2,3)$.

There has been extensive research regarding the diagnostic performance of ERUS in the staging of rectal cancer (4-6); however, its accuracy, reliability and validity remain controversial. The majority of the studies include $<100$ patients and represent only the initial institutional experience with this technique. There is currently no consensus regarding the performance of ERUS at each stage of rectal cancer. Furthermore, ERUS is highly dependent on examiner and institutional experience. Although most of these reports mention that there is a learning curve in performing and interpreting these 
Table I. Assessment of rectal wall invasion with ERUS (uT) vs. pathological examination (pT).

Pathological examination

\begin{tabular}{lcccccccc}
\cline { 2 - 4 } ERUS & pT1 (n) & pT2 (n) & pT3 (n) & pT4a (n) & pT4b (n) & Accuracy (\%) & Overstaged (\%) & Understaged (\%) \\
\hline uT1 & 2 & 1 & 2 & 1 & 0 & NA & NA & NA \\
uT2 & 1 & 25 & 29 & 3 & 0 & 43 & 2 & 55 \\
uT3 & 0 & 16 & 137 & 17 & 0 & 81 & 9 & 10 \\
uT4a & 0 & 7 & 26 & 48 & 0 & 59 & 41 & 25 \\
uT4b & 0 & 0 & 1 & 0 & 3 & 75 & \\
\hline
\end{tabular}

ERUS, endorectal ultrasonography; NA, data not available.

examinations, the available information regarding the length and steepness of that curve, or the early training required when using this modality, is currently limited. Thus, the purpose of our study, was to further assess the accuracy and learning curve of ERUS in the assessment of preoperative staging of rectal cancer in the Shanxi Province Tumor Hospital.

\section{Patients and methods}

Patient selection. The patients were selected based on four inclusion criteria as follows: i) all the patients required a positive biopsy report (rectal cancer); ii) all the patients underwent ERUS prior to treatment; iii) all the tumors were surgically excised and assessed histopathologically; and iv) ERUS diagnosis was directly compared with the histopathological findings. Patients with stenotic tumors and those undergoing incomplete examination were excluded.

All the rectal cancer patients presenting to the Shanxi Province Tumor Hospital between January, 2007 and March, 2010, were preoperatively assessed by ERUS. Any tumor within $12 \mathrm{~cm}$ of the anal verge on rigid sigmoidoscopy was defined as a rectal tumor, but only patients with histologically proven adenocarcinoma were included in the study. A total of 319 rectal cancer patients (175 men and 144 women), with a mean age of 59 years (range, 22-82 years) were included in this study. Of the 319 patients, 311 underwent radical surgery (abdominoperineal or low anterior resection) and 8 underwent transanal local excision.

The research protocols were approved by the Ethics Comittee of the Shan Xi province Tumor Hospital and all the patients provided written informed consent prior to enrolment.

Patient and physician classification. For the purposes of our analysis, the patients were divided into three groups, namely $\mathrm{A}, \mathrm{B}$ and $\mathrm{C}$, depending on whether the examination was performed between January and December, 2007 ( $n=38)$, between January and December, $2008(n=35)$, or between January, 2009 and March, $2010(n=146)$. Five physicians with no prior experience in ERUS performed the examinations. Two of the physicians performed only $15 \%$ of the examinations (47/319) and were excluded from our analysis of accuracy by physician. The remaining three physicians, namely D, E and F, performed 162, 64 and 46 examinations, respectively.
Procedure. After undergoing an enema $1 \mathrm{~h}$ prior to the ERUS preparation of the lower bowel, a rigid proctoscopy was performed on each patient to determine the distance of the tumor from the anal verge, the tumor size and the wall of the rectum on which the tumor was located. The patients were examined in the left lateral position. The ERUS was performed with a $7.5-\mathrm{MHz}$ scanner with a $360^{\circ}$ rotating ultrasonographic probe (Water Balloon Endo-P-Probe; Siemens AG, Munich, Germany). The probe was fitted with a rubber sheath and filled with degassed water to minimize acoustic artifacts and ensure acoustic coupling. The probe was lubricated using water-soluble gel and introduced under ultrasound control without the use of a rigid sigmoidoscope.

ERUS staging. Tumor invasion of the rectal wall was staged by the ultrasound tumor ( $\mathrm{T}$ staging) and node classification ( $\mathrm{N}$ staging) proposed by Hildebrandt and Feifel (7). According to this system and the 7th TNM classification (8), a uT1 lesion is confined to the mucosa and submucosa; a uT2 tumor penetrates the muscularis but is confined within the rectal wall, so that the outer hyperechoic layer remains intact; a uT3 tumor disrupts the outer hyperechoic layer, indicating invasion of the perirectal fat; a uT4a tumor penetrates to the surface of the visceral peritoneum; and a uT4b tumor directly involves surrounding organs or is adherent to other organs or structures. Normal, non-enlarged lymph nodes are similar in echogenicity to the hyperechoic perirectal tissues and, therefore, are not usually identified. Pathological lymph nodes, which were described as $\mathrm{uN1}$, were defined as circular structures $>5 \mathrm{~mm}$ in diameter, with a similar echogenicity to the tumor according to the description by Beynon et al (9). Nodes $<5 \mathrm{~mm}$ in diameter, which were defined as uN0, were considered to be normal or inflammatory.

Comparison of ERUS results with histopathological findings and statistical analysis. The ERUS results were compared with the postoperative histopathological findings for each resected specimen. The accuracy of $\mathrm{T}$ and $\mathrm{N}$ staging after each additional 20 patients was calculated for three physicians, namely D, E and F and the series of cumulative accuracies were displayed. The learning curve was indicated by a plateau of the cumulative accuracies. The comparison of the accuracies within the $\mathrm{T}$ and $\mathrm{N}$ staging results and between groups was performed using the $\chi^{2}$ test by SPSS statistical software, 
Table II. Assessment of lymph node metastasis with ERUS (uN) vs. pathological examination (pN).

\begin{tabular}{|c|c|c|c|c|c|c|}
\hline \multirow[b]{2}{*}{ ERUS } & \multicolumn{3}{|c|}{ Pathological examination } & \multirow[b]{2}{*}{ Accuracy (\%) } & \multirow[b]{2}{*}{ Overstaged (\%) } & \multirow[b]{2}{*}{ Understaged $(\%)$} \\
\hline & $\mathrm{pN} 1(\mathrm{n})$ & pNO (n) & Total (n) & & & \\
\hline uN1 & 85 & 35 & 120 & 71 & 29 & \\
\hline uN0 & 70 & 121 & 191 & 63 & & 37 \\
\hline Total & 155 & 156 & 311 & 66 & 11 & 23 \\
\hline
\end{tabular}

ERUS, endorectal ultrasonography.

Table III. Diagnostic accuracy of ERUS by physician during three different time periods.

\begin{tabular}{|c|c|c|c|c|c|c|c|}
\hline \multirow[b]{2}{*}{ Physicians } & \multirow[b]{2}{*}{ Patient no. } & \multicolumn{2}{|c|}{$\begin{array}{c}\text { Period A } \\
\text { (January-December, 2007) }\end{array}$} & \multicolumn{2}{|c|}{$\begin{array}{c}\text { Period B } \\
\text { (January-December, 2008) }\end{array}$} & \multicolumn{2}{|c|}{$\begin{array}{c}\text { Period C } \\
\text { (January, 2009-March, 2010) }\end{array}$} \\
\hline & & T stage (\%) & $\mathrm{N}$ stage $(\%)$ & T stage (\%) & $\mathrm{N}$ stage $(\%)$ & T stage (\%) & $\mathrm{N}$ stage $(\%)$ \\
\hline D & 162 & $55(12 / 22)^{\mathrm{a}}$ & $41(9 / 22)^{b}$ & $72(46 / 64)$ & $73(45 / 62)$ & $84(64 / 76)^{\mathrm{a}}$ & $81(58 / 72)^{\mathrm{b}}$ \\
\hline E & 64 & & & $43(10 / 23)$ & $52(12 / 23)$ & $66(27 / 41)$ & $63(26 / 41)$ \\
\hline F & 46 & $44(4 / 9)$ & $33(3 / 9)$ & $50(14 / 28)$ & $57(16 / 28)$ & $56(5 / 9)$ & $56(5 / 9)$ \\
\hline
\end{tabular}

${ }^{\mathrm{a}, \mathrm{b}} \mathrm{P}<0.01\left(\chi^{2}=6.65,13.27\right)$ by $\chi^{2}$ test. ERUS, endorectal ultrasonography.

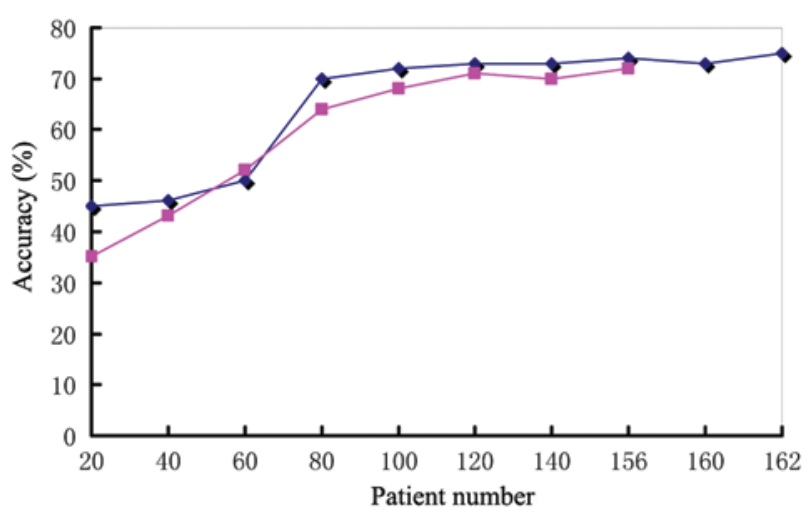

Figure 1. Learning curve of physician D.

version 19.0 (IBM Corp., Armonk, NY, USA). P<0.05 was considered to indicate a statistically significant difference.

\section{Results}

$T$ staging. The overall accuracy for $\mathrm{T}$ staging was $67 \%$, with $16 \%$ of the tumors being overstaged and $17 \%$ understaged. Of the 319 primary rectal tumors, 6 were uT1, 58 uT2, 170 uT3, 81 uT4a and 4 uT4b. The accuracy of T1 staging could not be meaningfully calculated due to the limited number of cases (Table I).

$N$ staging. The overall accuracy for $\mathrm{N}$ staging was $66 \%$; when incorrect, understaging tended to occur more frequently compared to overstaging (23 vs. 11\%, respectively) (Table II).

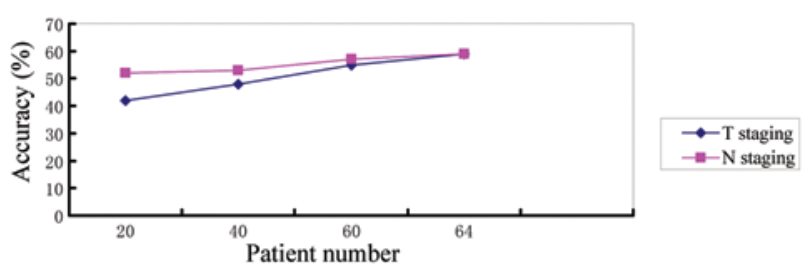

Figure 2. Learning curve of physician E.

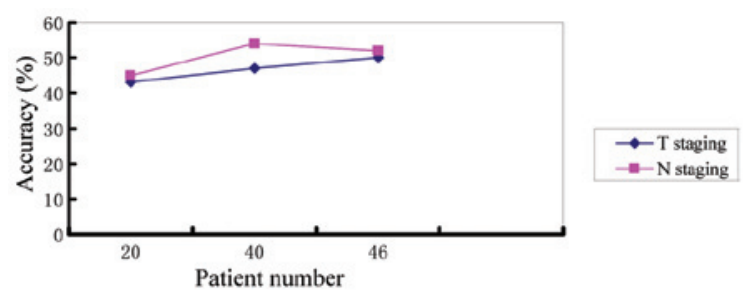

Figure 3. Learning curve of physician F.

A total of 311 nodes (nodal diameter, 2.0-33.4 cm) were examined with ERUS in the 311 patients who underwent radical surgery and 120 nodes were found to be positive for metastasis.

Diagnostic accuracy by physician during three different time periods. Physicians D, E and F completed the examination of 272 of the 319 cases. The total $\mathrm{T}$ and $\mathrm{N}$ staging accuracy of physicians D, E and F were 75 and $72 \%$; 59 and 59\%; and 50 and $52 \%$, respectively. The diagnostic accuracy of the physicians during time periods A, B and C, is shown in Table III. 
Learning curve. Two of the physicians performed only $15 \%$ of the examinations (47/319) and were excluded from our analysis of accuracy by physician. For the remaining three physicians, namely D, E and F, the accuracy of $\mathrm{T}$ and $\mathrm{N}$ staging after each additional set of 20 patients was calculated. The series of cumulative accuracies are displayed in Figs. 1-3.

\section{Discussion}

The modern treatment of rectal cancer is currently stage-oriented (10). Accurate preoperative clinical staging is crucial for planning treatment and determining the prognosis of patients on an individual basis. Over the last few years, transanal local excision has become an accepted surgical alternative for selected patients with rectal tumors confined within the rectal wall. Preoperative chemoradiation is the standard therapy in patients with advanced rectal cancer. The aim of preoperative treatment is to achieve downstaging and/or downsizing, with the intention to increase the resectability rate, enable sphinctersparing surgery, reduce local recurrence and possibly improve long-term survival $(11,12)$. However, the therapeutic strategies that attempt treatment by stage require a precise knowledge of the depth of tumor invasion of the rectal wall and of the presence of affected regional lymph nodes prior to the operation.

Over the last decade, although ERUS has become the most common diagnostic modality for local staging of rectal cancer, the accuracy of ERUS staging has been controversial. A review of the literature revealed that the accuracy of $\mathrm{T}$ staging with ERUS ranges between 81 and 95\% (13-15). These differences are mainly affected by factors such as the number of cases included in the study, experience with ERUS, patient selection (with or without radiation) and type of endorectal probe (2D and 3D). The number of patients in the majority of the studies is $<100$, although it may be higher for stage T3-4 cases. Garcia-Aguilar et al (13) reviewed the charts of 545 patients with rectal carcinomas staged by ERUS and compared the ERUS staging with the pathological findings based on the surgical specimens. The overall accuracy of $\mathrm{T}$ staging was $69 \%$, with $18 \%$ of the tumors being overstaged and $13 \%$ understaged.

In our study, the overall accuracy of T staging was $67 \%$, which is lower compared to that previously reported, but similar to that reported by Garcia-Aguilar, with $16 \%$ of the tumors overstaged and $17 \%$ understaged, which is similar to the percentages $(2.0-23.9 \%$ and $2.5-17.0 \%$, respectively) reported in the literature (16). ERUS accurately identified 2 of 3 pT1 tumors and $188(71 \%)$ of 264 pT3 or pT4 tumors that were candidates for preoperative chemoradiation therapy. However, the number of pT1 patients in those studies was inadequate to reach meaningful conclusions. Approximately $9 \%$ of $\mathrm{T} 2$ lesions may be overstaged due to a desmoplastic response, whereas $41 \%$ of $\mathrm{T} 3$ lesions may be understaged due to the presence of microscopic metastases. The poor endosonographic diagnosis of T2 and T4a tumors (43 and 59\%, respectively) is worse compared to that previously reported, except for the study of Sailer et al (17), who reported an accuracy of $41 \%$ in T2 tumors. The low accuracy of ERUS in the characterization of T2 tumors emphasizes the need to plan the final treatment after local excision based on the pathological rather than on the ultrasonographic stage. Yamashita et al (18) demonstrated that the overestimation of the extent of cancer invasion was increased in proportion to the degree of peritumoral inflammation, which makes hyperechoic layers in the rectal wall appear hypoechoic and may lead to disappearance of the outer hyperechoic layer, resulting in overstaging of T2 lesions. In our study, the tumor-induced inflammation was falsely diagnosed on preoperative ERUS as bladder invasion (uT4b). During the operation, we observed adhesion formation between the rectum and the posterior wall of bladder and resected that part of the bladder wall. After surgery, the pathological examination confirmed chronic inflammation of the bladder wall, whereas the tumor only invaded perirectal fat tissue (pT3). When initially applying this technique, due to the operator's limited experience, the water-filled balloon was quite sizeable, applying excessive pressure on the intestine, making it difficult to distinguish the rectal wall layers. As seen in Table III, with increasing experience, the accuracy of physician D improved, with a statistically significant difference in $\mathrm{T}$ staging accuracy between time periods $\mathrm{A}$ and $\mathrm{C}\left(\chi^{2}=6.65, \mathrm{P}<0.01\right)$.

Lymph node metastasis was found to be an independent prognostic factor for patient survival and local disease recurrence. According to the literature, the accuracy for detection of malignant lymph nodes ranges between 64 and 84\% (13-15). In a meta-analysis, Bipat et al (19) reported that the sensitivity of ERUS, CT and MRI for lymph node metastasis was 67, 55 and $66 \%$, respectively, whereas their specificity was 78,74 and $76 \%$, respectively, indicating that there were no significant differences among ERUS, CT and MRI in nodal staging. In our study, the overall accuracy of $\mathrm{N}$ staging was $66 \%$, which is lower compared to that previously reported, with $11 \%$ of the tumors being overstaged and 23\% understaged. Lymph node staging is difficult and challenging. Several studies adopted $5 \mathrm{~mm}$ as a cut-off size for discriminating between malignant and benign nodes; however, nodal size is not a reliable discriminator. Imaging is mainly based on the size of the lymph nodes, but cannot identify micrometastases. In a study of 424 surgical rectal cancer specimens including a total of 12,759 nodes, the mean nodal diameter was $3.34 \mathrm{~mm}$ and the mean diameter of metastasis was $3.84 \mathrm{~mm}$ (20). In another pathological study of 698 lymph nodes, 70 of 132 (53\%) nodes containing metastases were $<5 \mathrm{~mm}$ in diameter (21). In our study, the minimum diameter of the lymph nodes identified with ultrasound was $2.0 \mathrm{~mm}$ and the maximum $33.4 \mathrm{~mm}$. A total of $71 \%(85 / 120)$ lymph nodes $>5 \mathrm{~mm}$ were found to be metastatic, as were $53 \%(10 / 19)$ of those sized $<4 \mathrm{~mm}$. Thus, diameter alone cannot determine the presence of metastasis in a lymph node. In addition, benign nodal hyperplasia was a common finding. The overstaging of lymph nodes is primarily caused by the presence of reactive enlarged lymph nodes that may be misdiagnosed as malignant. In addition, small blood vessels and the seminal vesicles are occasionally mistaken for metastatic lymph nodes (22). It was previously demonstrated that endoscopic ultrasound-guided fine-needle aspiration and $3 \mathrm{D}$ transrectal ultrasound may improve $\mathrm{T}$ and $\mathrm{N}$ staging accuracy $(23,24)$.

Although ERUS is an operator-dependent procedure and its results are closely associated with operator experience, only a limited number of studies in the literature provide information on the learning curve. One such study by Orrom et al (25) reported on 77 rectal cancer patients assessed ultrasonically at 
the University of Minnesota. In that study, the patients were divided into three consecutive groups as follows: group A, 27 patients in the first 15 months; group B, 30 patients in the next 10 months; and group C, 20 patients in the last 6 months. The $\mathrm{T}$ staging accuracy improved from $58 \%$ in the first group to $77 \%$ in the second group and, finally, to $95 \%$ in the third group. The difference between the accuracies of the first and second group was statistically significant, as was the difference in the accuracies between the first and third group. No conclusions were drawn with regards to a learning curve for $\mathrm{N}$ staging.

A more recent study from the Colorectal Surgical Society of Australia and New Zealand was published by Morris et al (26). A prospective study of ERUS for staging rectal cancer by a single surgeon from commencement of consultant practice was performed. The results were compared over three time periods: the first within a single year, followed by 2- and 3-year periods. A total of 233 cases were assessable for T staging and 142 for $\mathrm{N}$ staging. The overall accuracy was $82 \%$ for $\mathrm{T}$ staging and $73 \%$ for $\mathrm{N}$ staging. The accuracy for $\mathrm{T}$ and $\mathrm{N}$ staging did not change significantly over the three time periods $(\mathrm{P}>0.05)$. The authors suggested that accuracy does not improve with further experience; however, an ERUS accreditation scheme should be established for future trainees. As shown in Fig. 1, the T staging accuracy of physician D increased from $45 \%$ (20 cases) to $70 \%$ (80 cases) after the curve had stabilized and the difference was significant $(\mathrm{P}<0.05)$. The $\mathrm{N}$ staging accuracy increased from 35 to $64 \%$ and the difference was also statistically significant $(\mathrm{P}<0.05)$. After 80 cases, the staging accuracy of physician $\mathrm{D}$ reached a plateau. In Figs. 2 and 3, the difference between the accuracy of physician $\mathrm{B}$ and $\mathrm{C}$ and was not statistically significant $(\mathrm{P}>0.05)$; however, with the increase in the number of patients examined, their accuracies improved. Our experience suggests that there is a learning curve about ERUS.

This study had certain limitations. First, this was a retrospective, observational study conducted by a single center. Second, all the examinations were performed by five physicians. The strength of this study lies with the fact that we performed more examinations (319 patients), which were divided into three groups depending on the visiting time. Although in the abovementioned studies the overall accuracy appears to be better compared to ours, there are a few methodical differences between the studies. First, five board-certified physicians performed all the assessments in our study, whereas in the study by Morris et al (26), a single consultant radiologist conducted all the examinations. Second, the five physicians in our study had no prior experience with ERUS. As shown in Table III, the T staging accuracy of physician D increased from $55 \%$ (period A) to $84 \%$ (period C) and the difference was statistically significant $(\mathrm{P}<0.01)$. The $\mathrm{N}$ staging accuracy increased from 41 to $81 \%$ and the difference was also statistically significant $(\mathrm{P}<0.01)$. However, the number of patients examined by physicians $\mathrm{E}$ and $\mathrm{F}$ was limited; therefore, there was not statistically significant difference in their staging accuracy between period $\mathrm{B}$ and period $\mathrm{C}(\mathrm{P}>0.05)$. Third, the five-layer rectal wall model was the standard interpretation throughout our study. The three-layer model was used for the first two groups in the Minnesota study, with the five-layer model used for the last group of patients. Therefore, in the study by Orrom et al (25), none of the groups were interpreted with consistent criteria, making conclusions on the learning curve for ERUS difficult.
We sought to overcome these problems by using consistent parameters. Of note, although constant definitions are preferable, there is a debate over the number of definable layers $(7,9)$. The muscularis propria of the rectal wall has been suggested as being a two- or three-layer structure $(7,27)$. Therefore, uT2 lesions may be subdivided. However, as this would not significantly affect clinical management, we did not consider it was important to make such a distinction.

ERUS is a valuable diagnostic tool. Provided that the examiner completes a certain number of examinations ( $~ 80$ cases), accumulates experience and masters the technique, ERUS may be used for the preoperative staging of rectal cancer. In conclusion, there is a learning curve in the preoperative staging of rectal carcinoma by ERUS. However, the results of ERUS during the learning process must be interpreted with caution for clinical decision making. The combination of ERUS with other diagnostic methods may lead to a more accurate prediction of the local stage in rectal cancer patients.

\section{References}

1. Nedrebø BS, Søreide K, Eriksen MT, Kvaløy JT, Søreide JA and Kørner H: Excess mortality after curative surgery for colorectal cancer changes over time and differs for patients with colon versus rectal cancer. Acta Oncol 52: 933-940, 2013.

2. Samee A and Selvasekar CR: Current trends in staging rectal cancer. World J Gastroenterol 17: 828-834, 2011.

3. Fernández-Esparrach G, Ayuso-Colella JR, Sendino O, et al: EUS and magnetic resonance imaging in the staging of rectal cancer: a prospective and comparative study. Gastrointest Endosc 74: 347-354, 2011

4. Cârţână ET, Pârvu D and Săftoiu A: Endoscopic ultrasound: current role and future perspectives in managing rectal cancer patients. J Gastrointestin Liver Dis 20: 407-413, 2011.

5. Kav T and Bayraktar Y: How useful is rectal endosonography in the staging of rectal cancer? World J Gastroenterol 16: 691-697, 2010.

6. Puli SR, Bechtold ML, Reddy JB, et al: Can endoscopic ultrasound predict early rectal cancers that can be resected endoscopically? A meta-analysis and systematic review. Dig Dis Sci 55: 1221-1229, 2010

7. Hildebrandt U and Feifel G: Preoperative staging of rectal cancer by intrarectal ultrasound. Dis Colon Rectum 28: 42-46, 1985.

8. Edge S, Byrd DR, Compton CC, Fritz AG, Greene FL and Trotti A (eds): AJCC Cancer Staging Manual. 7th edition. Springer, New York, 2010.

9. Beynon J, Foy DM, Temple LN, Channer JL, Virjee J and Mortensen NJ: The endoscopic appearances of normal colon and rectum. Dis Colon Rectum 29: 810-813, 1986.

10. Ayuso Colella JR, Pagés Llinás M and Ayuso Colella C: Staging rectal cancer. Radiologia 52: 18-29, 2010 (In Spanish).

11. Popek S and Tsikitis VL: Neoadjuvant vs. adjuvant pelvic radiotherapy for locally advanced rectal cancer: which is superior? World J Gastroenterol 17: 848-854, 2011.

12. Sermeus A, Leonard W, Engels B and De Ridder M: Advances in radiotherapy and targeted therapies for rectal cancer. World J Gastroenterol 20: 1-5, 2014.

13. Garcia-Aguilar J, Pollack J, Lee SH, Hernandez de Anda E, Mellgren A, Wong WD and Finne CO, Rothenberger DA and Madoff RD: Accuracy of endorectal ultrasonography in preoperative staging of rectal tumors. Dis Colon Rectum 45: 10-15, 2002.

14. Nesbakken A, Lovig T, Lunde OC and Nygaard K: Staging of rectal carcinoma with transrectal ultrasonography. Scand J Surg 92: 125-129, 2003.

15. Mackay SG, Pager CK, Joseph D, et al: Assessment of the accuracy of transrectal ultrasonography in anorectal neoplasia. Br J Surg 90: 346-350, 2003.

16. Lin S, Luo G, Gao X, et al: Application of endoscopic sonography in preoperative staging of rectal cancer: six-year experience. J Ultrasound Med 30: 1051-1057, 2011.

17. Sailer M, Leppert R, Bussen D, Fuchs KH and Thiede A: Influence of tumor position accuracy of endorectal ultrasound staging. Dis Colon Rectum 40: 1180-1186, 1997. 
18. Yamashita Y, Machi J, Shirouzu K, Morotomi T, Isomoto H and Karegawa T: Evaluation of endorectal ultrasound for the assessment of wall invasion of rectal cancer: report of a case. Dis Colon Rectum 31: 617-623, 1998.

19. Bipat S, Glas AS, Slors FJ, Zwinderman AH, Bossuyt PM and Stoker J: Rectal cancer: local staging and assessment of lymph node involvement with endoluminal US, CT, and MRI - a meta-analysis. Radiology 232: 773-783, 2004.

20. Dworak O: Morphology of lymphnodes in the resected rectum of patients with rectal carcinoma. Pathol Res Pract 187: 1020-1024, 1999.

21. Monig SP, Baldus SE, Zirbes TK, et al: Lymph node size and metastatic infiltration in colon cancer. Ann Surg Oncol 6: 579-581, 1999.

22. Kruskal JB, Kane RA, Sentovitch SM and Longmaid HE: Pitfalls and sources of error in staging rectal cancer with endorectal US. Radiographics 17: 606-626, 1997.

23. Knight CS, Eloubeidi MA, Crowe R, et al: Utility of endoscopic ultrasound-guided fine-needle aspiration in the diagnosis and staging of colorectal carcinoma. Diagn Cytopathol 41: 1031-1037, 2013 .
24. Tan KK and Tsang CB: Staging of rectal cancer - technique and interpretation of evaluating rectal adenocarcinoma, uT1-4, N disease: 2D and 3D evaluation. Semin Colon Rectal Surg 21: 197-204, 2010

25. Orrom WJ, Wong WD, Rothenberger DA, Jensen LL and Goldberg SM: Endorectal ultrasound in the preoperative staging of rectal tumors: a learning experience. Dis Colon Rectum 33: 654-659, 1990.

26. Morris OJ, Draganic B and Smith S: Does a learning curve exist in endorectal two-dimensional ultrasound accuracy? Tech Coloproctol 15: 301-311, 2011.

27. KatsuraY, Yamada K, Ishizawa T, Yoshinaka H and Shimazu H: Endorectal ultrasonography for the assessment of wall invasion and lymph node metastasis in rectal cancer. Dis Colon Rectum 35: 362-368, 1992. 